CAHIERS DE

NARRATOLOGIE
Cahiers de Narratologie

Analyse et théorie narratives

$16 \mid 2009$

Images et récits

\title{
Hors-limite: Equipo Crónica ou l'éclatement du message narratif
}

Fabrice Parisot

\section{(2) OpenEdition}

Journals

Édition électronique

URL : https://journals.openedition.org/narratologie/1042

DOI : 10.4000/narratologie.1042

ISSN : 1765-307X

Éditeur

LIRCES

Référence électronique

Fabrice Parisot, «Hors-limite: Equipo Crónica ou l'éclatement du message narratif », Cahiers de Narratologie [En ligne], 16 | 2009, mis en ligne le 25 mai 2009, consulté le 28 juin 2022. URL : http:// journals.openedition.org/narratologie/1042 ; DOI : https://doi.org/10.4000/narratologie.1042

Ce document a été généré automatiquement le 30 septembre 2020.

\section{(c) (i) (9)}

Cahiers de Narratologie - Analyse et théorie narratives est mis à disposition selon les termes de la licence Creative Commons Attribution - Pas d'Utilisation Commerciale - Pas de Modification 4.0 International. 


\title{
Hors-limite: Equipo Crónica ou l'éclatement du message narratif
}

\author{
Fabrice Parisot
}

1 S'il y a bien un type de peinture qui semble avoir plein droit de cité dans ce volume consacré aux limites du récit, c'est sans nul doute celle du collectif espagnol Equipo Crónica.

2 S'inscrivant en effet dans une période artistique de crise marquée par l'hégémonie de la peinture informelle et abstraite, les deux peintres valenciens Rafael Solbés et Manolo Valdés vont, au milieu des années 60 , rompre avec l'orthodoxie de la peinture académique aseptisée en déconstruisant les messages narratifs traditionnels et en proposant un langage pictural nouveau destiné à dépasser les limites d'une peinture engoncée dans un certain académisme, plongée dans le subjectivisme et engluée dans l'introspection et promener un regard critique sur la société espagnole du franquisme finissant.

3 C'est donc à une véritable rénovation, pour ne pas dire à une nécessaire révolution, des codes picturaux à laquelle les deux artistes vont se livrer afin d'aborder et de développer plastiquement les problèmes inhérents à la situation sociale, politique, économique et culturelle d'une période historique particulière marquée par une dictature pour le moins peu encline aux manifestations artistiques originales et novatrices, mais soucieuse cependant de son image, notamment sur la scène internationale.

Cependant on aurait tort de croire que cette déconstruction des codes traditionnels, que cette infraction qui conduit souvent à une fragmentation de l'image, à une vision kaléidoscopique des événements se limitât ou se bornât à une critique virulente, à une chronique ironique d'une réalité quotidienne placée sous le signe de la violence et des privations des libertés ou à un simple témoignage. En effet, par-delà les aspects purement idéologiques que les toiles abordent sans complaisance, les deux peintres se livrent également à une réflexion esthétique qui tend à interroger l'image et à s'interroger sur la création artistique, sur la pratique de la peinture et sur la peinture elle-même. Et, on 
l'aura compris, à s'interroger sur la fonction de l'art et sur les rapports que ce dernier entretient avec la société.

5 Peinture métapicturale donc, peinture engagée certes, mais une peinture en tout état de cause qui va s'efforcer de dépasser les formes que prend traditionnellement le récit iconique canonique. Ce sont par conséquent ces limites qu'Equipo Crónica semble avoir manifestement transgressées et - nous le verrons - allègrement franchies que nous allons à présent délimiter, dans ses aspects les plus singuliers, afin de déterminer les implications esthétiques et idéologiques que suppose une telle entreprise.

6 En 1964, à l'occasion du XVIème Salon de la Jeune Peinture organisé à Paris par Arroyo, Aillaud et Recalcati, les peintres valenciens Rafael Solbés, Manolo Valdés et Joan Antoni Toledo, issus des groupes Estampa Popular et Crónica de la Realidad décident, à partir des propositions théoriques de Tomas Llorens, de former le collectif Equipo Crónica. Bien que lors de cette exposition, chacun des trois peintres présentât des œuvres séparément, la volonté de travailler à un projet plastique commun selon les mêmes présupposés esthétiques et de réaliser des œuvres à six mains, puis très rapidement à quatre mains (Toledo abandonnera très tôt le collectif) se fit jour dans l'optique de rénover le panorama artistique espagnol.

7 En 1965, peu après leur participation à ce Salon, les trois peintres exposeront, à Paris toujours, une oeuvre réalisée cette fois-ci conjointement et intitulée Une Passion dans le désert. Il s'agissait d'une oeuvre narrative basée sur un court récit de Balzac. En septembre de la même année, les mêmes artistes exposent Vivre ou laisser mourir : la fin tragique de Marcel Duchamp, une série de 10 tableaux qui se présentent comme le bref récit d'une action: l'assassinat de Marcel Duchamp par les 3 auteurs, narrée dans un style volontairement dépersonnalisé.

On comprendra dès lors aisément que la constitution du Collectif s'inscrit, au niveau esthétique, dans une démarche qui vise à rejeter le courant informel, l'abstraction géométrique et l'expressionnisme abstrait alors en vogue dans l'Espagne du franquisme finissant et à réagir contre les différents styles qui dominent durant la période dite de l'après-guerre afin d'introduire une touche de réalisme et une palette plus ou moins colorée de critique sociale, mais également politique, culturelle, historique et économique.

9 Il faut bien reconnaître que jusqu'à la naissance, au début des années 60 , d'un certain nombre de groupes comme Estampa Popular (1960) dont la finalité, pour rompre avec le passé informel, était de réduire la distance entre l'art et la société par la création d'images compréhensibles, populaires et économiquement accessibles ou comme Crónica de la Realidad (1961) qui, tout en se rapprochant de la Nouvelle Figuration proposait un mélange d'art informel expressionniste et de Pop Art dans le but de proposer une peinture qui concilie art et réalité sociale, toute la création artistique en Espagne est alors dominée par l'art informel (Tapiés, Millares, Saura) qui apparaît aux yeux de la critique comme un répertoire de gestes, de textures et de matériaux qui donnent une apparence de modernité mais qui ramènent à des notions fondamentalement académiques, voire à un certain conservatisme. Quant à l'abstraction expressionniste, apanage du groupe $\mathrm{El}$ Paso (1957-1960), elle suppose une peinture abstraite de caractère subjectif, baroque et existentiel, inspirée de l'automatisme surréaliste tandis que l'abstraction géométrique se fonde sur la prédominance de la raison, de l'ordre et de la discipline comme le prônent les groupes Parpallo et Equipo 57. 
10 Equipo Crónica, en réaction à ce type de peinture, va donc s'efforcer de dépasser, voire de contester, les formes que prenaient alors le récit iconique et, dans le même temps, de déroger à l'orthodoxie du discours canonique, bouleversant les implications idéologiques et esthétiques pour donner naissance à un type de création qui, selon la critique, constitue une étape fondamentale dans l'histoire de l'art espagnol.

11 Par ailleurs, le débat sur la relation entre l'art et la société, l'éclosion du Pop Art, le développement des médias, l'irruption de la pensée marxiste dans l'analyse de l'art, les apports du structuralisme et de la sémiotique allaient donner, dans les années 60 , de nouvelles armes idéologiques, esthétiques et politiques dans le cadre d'un régime dictatorial diminué. Face au subjectivisme de l'art informel allait désormais s'imposer le travail d'équipe.

12 Au-delà d'une activité de groupe, la pratique en équipe signifiait obligatoirement la mise en place d'un langage extrêmement objectif, accompagné d'une cohérence formelle et conceptuelle. Cela explique certainement qu'Equipo Crónica ait eu une manière tout à fait particulière de configurer les plans picturaux, de distribuer les pigments, d'organiser les figures, d'ordonner les citations iconographiques et de développer les thèmes. Pour ce faire, Equipo Crónica va revendiquer un pacte avec la réalité pour donner à la peinture une raison d'être sociale et se livrer à une interprétation particulière du réalisme Pop en empruntant aux médias les procédés techniques qui permettent la déformation optique ou la répétition traitées avec une ironie critique adaptée à la situation politique espagnole.

13 Les cinq années qui s'étendent de 1964 à 1969 - période d'apprentissage en commun vont être principalement consacrées à acquérir et à mettre en pratique tous les éléments constitutifs d'un discours original tendant à dépasser les moyens de représentations traditionnels et stéréotypés et à éviter tout symbolisme inexpressif. Elles apparaissent comme un moment clé de la gestation du groupe car elles permettent d'avoir une vision complète - ou presque - des techniques qui seront utilisées tout au long de la production des deux peintres jusqu'en 81, même si après 1968 les tableaux réalisés auront tendance soit à se compliquer soit, au contraire, à se simplifier. Elles apparaissent également comme le moment privilégié où Solbés et Valdés, s'écartant des utopies de l'avant-garde historique, vont dépouiller la peinture de ses limites, faire sauter les carcans et les verrous dans lesquels la classe dominante a l'habitude de l'enfermer depuis des siècles. Dès lors, Equipo Crónica va déconstruire les codes traditionnels de la peinture et va montrer les possibilités extrêmes (acception du mot limite) que peut offrir et que doit, selon eux, revêtir la création artistique.

14 Les deux artistes, il convient de le rappeler, se situent dans la perspective de la critique sociale, déjà abordée au préalable par les autres peintres d'Estampa Popular et de Crónica de la Realidad. Le caractère de témoignage ou de reportage de leur oeuvre se manifeste alors dans la représentation des problèmes sociaux de la péninsule - l'émigration, les manifestations d'étudiants... - et/ou dans la représentation de l'actualité internationale comme la guerre du Vietnam, à partir d'images diffusées par la presse et la télévision¹.

De façon extrêmement novatrice, dépassant largement les cadres de la peinture traditionnelle, Solbés et Valdés vont avoir recours à l'image médiatique qui permet une multiplicité de points de vue visuels et, en même temps, suscite une réflexion sur la manipulation de l'image. Rien d'étonnant ou presque puisque les deux peintres vivaient dans une société qui assimilait et, dans le même temps, se soumettait aux nouveaux 
moyens de communication de masse, dont ils allaient abondamment tirer profit pour élaborer leurs chroniques.

Leurs premières oeuvres recueillent et accueillent des images qui renvoient essentiellement au blanc et noir des medias et, au point de vue photographique, propre au reportage ainsi qu'à des séquences semblables à celles des films avec des cadrages progressifs des effets de zoom, des montages de plans anachroniques; d'autres fois, les images passent par le filtre de miroirs ou de prismes qui les déforment. Il s'agissait dans tous les cas de provoquer une rupture dans la logique de la lecture traditionnelle, un glissement du processus narratif.

En plus des déformations, des distorsions et des effets de zoom tels qu'on peut les observer dans KKK (1965), Las caretas (1965), El banquero (1965), La demagogia (1966), Deformacion profesional (1066), Desarrollo historico (1966) ou General (1066) pour ne citer que quelques exemples significatifs, Equipo Crónica va développer une narration qui s'exprime dans la fragmentation de l'image (Estudiantes, Baile, Ha llegado su excelencia!, tous trois de 1965), la répétition - ou fausse répétition - d'une figure à l'intérieur de compartiments ou de cases (La noticia, Los ingredientes, El disparo, également de 1965), procédés caractéristiques du Pop Art, essentiellement empruntés à l'appareil formel d'Andy Wharol, mais au milieu de laquelle on crée une rupture en insérant un élément provocateur : dans América, América (1965) par exemple, Solbés et Valdés utilisent en la répétant, dans une série de cases identiques, la figure de Mickey, une des images typiques et topiques de la culture aux Etats-Unis, symbole de bonne humeur, emblème du bonheur, comme image - référent de la société de consommation. Mais, de façon inattendue, ils introduisent presque à la fin de la lecture, quand le regard ne s'y attend pas ou ne s'y attend plus, une image violente - le champignon de l'explosion atomique contre le Japon - qui charge le tableau d'un sens critique. C'est-à-dire qu'au lieu de la sensation d'angoisse qui naît habituellement du système répétitif comme l'indique Werner Spies à propos de l'artiste nord-américain, Solbés et Valdés créent un processus d'ennui fortement contrarié en fin de parcours narratif par l'impact que provoque cette rupture.

Très tôt et très vite, les deux peintres vont donc s'approprier le système de référence des moyens de communication, de l'appareil formel de répétition, de la banalisation de l'image ainsi que des techniques propres au Pop Art. Solbés et Valdés utilisent ainsi des techniques mécaniques, comme celle du dessin et de la sérigraphie. La stratégie adoptée - et toujours adaptée à la situation traitée - pour harmoniser le contenu critique et la forme POP consiste à choisir une photo d'un événement parue dans la presse : la guerre, une revue de troupes, une visite officielle ou une manifestation. Ils isolent l'un des personnages de cette iconographie et ils le soumettent à des changements d'échelle, ils effectuent des effets de zoom, des déformations progressives au long d'une série de compartiments comme dans Déformation professionnelle où un soldat nord-américain se transforme progressivement en guérillero vietnamien ou dans Vietnam où l'image fragmentée d'un soldat américain s'oppose à celle d'une famille du pays asiatique. Ces toiles se donnent alors à lire comme des critiques du militarisme américain. D'autre fois enfin, les figures et les fonds subissent divers types de manipulation: verticalité et horizontalité tronquées, effets de distorsion convexes ou concaves. Les exemples abondent...

Déconstruire l'image et en dévier le sens est évidemment l'un des mécanismes privilégiés employé dans un souci d'objectivité face au langage médiatique, à sa diffusion et à sa réception dans un pays replié sur lui-même qui ne laisse filtrer que les informations 
provenant des Etats Unis, notamment en ce qui concerne le conflit au Vietnam. L'objectif consistait à obliger le spectateur à lire autrement toute l'information que diffusaient alors tous les moyens de communication.

A partir de 1966-67, Equipo Crónica abandonnera le procédé de la compartimentation et choisira plutôt d'introduire dans ses compositions des citations de la peinture espagnole classique qu'ils confrontent à divers éléments émanant de la nouvelle société espagnole de consommation. Une grande partie d'ailleurs des sources de l'iconographie des objets présents dans les toiles provient du monde de la publicité - autre support souvent utilisé - plus précisément de revues de l'époque qui regorgeaient d'encarts publicitaires. De plus, dans les représentations des nouvelles technologies (ordinateurs, machines à laver $\left.{ }^{2}, . ..\right)$, Solbés et Valdés conservent fidèlement le blanc et le noir des revues de référence qui forment alors un contraste étonnant avec les couleurs des divers personnages tirés de la peinture espagnole des XVIème, XVIIème et XVIIIème siècles. Ces objets ne renvoient donc pas à des formes esthétiques, mais doivent plutôt être perçus comme des éléments immergés dans un contexte social, politique et historique donné. Dans Bodegón español par exemple, la Famille de Charles IV cohabite avec la représentation peu orthodoxe, loin s'en faut, d'une nature morte traitée à la manière des artistes pop nord-américains. Mais le simple fait d'introduire la classe dominante dans un espace prosaïque constitue une critique évidente : l'acceptation de la société de consommation érigée en modèle de comportement par la classe dirigeante.

21 Mais la présence de citations de peintres ou de peintures ne se cantonnera pas exclusivement à cette période-là puisque toute la production d'Equipo Crónica se caractérisera par la présence d'images empruntées à l'Histoire de l'art. Nombre de leurs compositions s'inspirent en effet de la peinture espagnole du Siècle d'or ou de la peinture contemporaine. Mais, il est vrai que ces emprunts sont à tout coup insérés dans des représentations qui ont pour cadre la société espagnole des années 60 à 80 . Ainsi abondent par exemple des références directes à Velázquez, à Zurbarán, à Ribera, à Goya, à Picasso, à Miró mais également à Kandinsky, à Magritte, à Grosz, à Kirchner, et à bien d'autres encore.

Enfin, Equipo Crónica s'est également essayé au cours de ces années d'apprentissage aux processus associatifs qui consistent à manipuler des citations simples comme dans la toile El tigre de papel où l'image stéréotypée est associée à des soldats afin d'aboutir à une allégorie de l'agression américaine auVietnam.

23 Mais il est évident que les apports des deux peintres en matière d'innovation et d'élaboration d'un nouveau code pictural qui permette de mettre en évidence un contexte social, politique et historique particulier et de rompre avec l'orthodoxie d'un langage formel conventionnel ne s'arrête pas à ces éléments, pour la plupart hérités du POP ART.

Equipo Crónica va également s'intéresser à d'autres moyens de communication comme la radio mais également, la bande dessinée ou le cinéma. A tel point d'ailleurs que les séries - dont il sera question plus avant- seront construites comme des séquences cinématographiques, comme plusieurs plans d'une même histoire, de sorte que les toiles apparaissent à la fois comme un tout autonome mais, dans le même temps, comme un fragment d'histoire. Ce sera principalement le cas dans la série intitulée Serie negra (1972) où les références à la présence du cinéma seront les plus explicites. Les deux personnages du tableau intitulé El yeso dérivent par exemple d'une image empruntée à un dictionnaire de thriller où Sterling Hayder et Elisha Cook jouent le rôle de gangsters dans The Killing de Stanley Kubrick. De la même façon, le tableau intitulé Los clasicos renvoie aux westerns 
spaghettis et aux films dits de l'Ouest. Mais l'exemple certainement le plus éclairant de cette extension de la peinture au cinéma réside dans le tableau intitulé Ve y oir(1974) dans lequel un appareil de radio se superpose à une photo tirée du tableau $\mathrm{El}$ garrote vil de Ramon Casas qui a été soumise à un processus de répétition assimilée à une séquence filmique.

Quant à la bande dessinée, elle est notamment présente dans la composition de toilesphylactères (El realismo socialista y el pop art en el campo de batalla) mais surtout à travers l'inclusion, voire l'intrusion, dans un certain nombre de tableaux, d'un personnage récurrent, El Guerrero del Antifaz, comme c'est le cas dans la série intitulée Guernica, et, de façon éclatante, dans le tableau El intruso. Mais, on le retrouve également dans Factoria (1969) et dans El banquete (1969)

Mais, par-delà ces apports externes qui rendent compte d'une volonté de créer un langage pictural nouveau qui déborde largement les limites de la peinture traditionnelle, les deux peintres valenciens se sont essayés à d'autres procédés techniques qui ressortissent directement de la peinture comme le collage ou le photomontage et qui s'inscrivent dans la même perspective, d'autant plus que les collages d'Equipo Crónica sont souvent des collages en trompe l'œil et non de véritables collages. A la différence du dadaïsme et du cubisme analytique qui utilisaient ce procédé pour rompre avec le naturalisme, montrer des pratiques quotidiennes, introduire des objets et des couleurs indépendantes du coup de pinceau subjectif et condamner le pouvoir mystificateur de l'artiste, opérant ainsi une rupture avec les codes conventionnels, Equipo Crónica, qui a parfaitement digéré cet héritage, pousse - par le procédé du trompe l'œil - les limites du collage encore plus loin et en fait un élément stratégique au moment de réfléchir sur la nature profonde de la peinture et de ses implications dans le traitement de la représentation de la réalités. Quant au concept de photomontage, on le retrouve dans un certains nombre de toiles réalisées notamment en 1974 et 1975 comme dans Rupture 1, Rupture 2 et Renau fotomontador, où la présence de ciseaux et d'images d'œuvres emblématiques découpées visent non seulement à enrichir la narration autour du thème de la récupération de certaines toiles mythiques par la culture dominante qui les dépouille ainsi de leur contenu critique ou subversif mais également à démythifier l'image du créateur et à désacraliser la peinture en tant qu'art.

Enfin, certaines toiles peuvent prendre la forme d'œuvres sans limites dans la mesure où elles condensent dans un même espace pictural une multitude de procédés comme dans $E l$ balcon par exemple où s'entremêlent des couleurs planes, assez neutres, des images tirées d'archives ou des moyens de communication, des personnages de bande dessinée ou de tableaux, notamment de La rendicion de Breda de Velázquez (avec de surcroît une inversion de l'image pour donner plus de crédibilité à la narration). Mieux encore, à toutes ces références mises en relation, organisées en réseau vient s'ajouter la figure centrale de Velázquez dont la taille crée une rupture par rapport à l'ensemble afin de renvoyer, visuellement et conceptuellement, à l'image du Caudillo à son balcon et permet une allégorie du pouvoir espagnol sans frontière de temps. A travers cette toile, on assiste alors à la déconstruction linéaire du discours pour créer un autre type de discours qui puisse se laisser appréhender selon divers niveaux de lecture.

Tous ces procédés qui, on en conviendra aisément, déconstruisent le message iconique traditionnel et dépassent largement les limites de la représentation conventionnelle, constituant dans un certain sens une véritable révolution du langage pictural, sont avant tout mis au service d'une critique politique et sociale puisque les références socio- 
historiques présentes dans les toiles embrassent un large éventail : la classe dirigeante, les oligarchies, le système capitaliste, l'impérialisme et ses ingérences, la guerre du Vietnam, le Che, les grèves, les manifestations, les militaires, même si, peu à peu, les toiles reflètent davantage et prioritairement les évolutions d'une société de consommation en plein développement. La tâche que s'assigne Equipo Crónica est avant tout celle d'une critique sociale acerbe, d'un reportage sur le vif, d'un témoignage plastique sur une réalité prosaïque.

Toutes ces innovations formelles qui embrassent un large champ de recours picturaux visent donc à une incursion dans la réalité culturelle et politique et à rendre compte d'un état des lieux de la situation espagnole. Mais si la détermination politique est présente dès les premières œuvres et le restera durant presque toute la production des deux peintres, on peut apprécier dans leur trajectoire un éloignement progressif de la volonté ingénue de re-présenter le monde de manière immédiate, une prise de conscience croissante que tout réalisme est construction, qu'il n'y a pas à accepter les formes de représentation historiquement codifiées comme réalistes pour parler du monde et que, par conséquent, il y a d'autres langages possibles, politiquement plus efficaces, pour critiquer la réalité. L'art révolutionnaire, à tout le moins porteur d'une forte charge subversive, ne peut se limiter, comme le pensait Brecht, à transmettre son message à travers des voies établies, c'est-à-dire qu'il ne peut pas prétendre enfermer dans une forme conventionnelle un contenu progressiste. C'est pourquoi la rénovation du langage pictural que mena à bien Equipo Crónica s'inscrit dans la logique de la nature combative de son œuvre puisque la tendance politique d'une œuvre ne se manifeste pas seulement dans son message ou dans les opinions de son auteur mais dans la propre forme artistique qui sert nécessairement de support idéologique.

On constate donc qu'après la période de l'art informel et de l'abstraction qui supposaient une introspection pour le moins peu subversive, Solbés et Valdés commencent à regarder le monde qui les entoure : une société marquée par l'industrialisation, la promotion du tourisme et le développement des nouvelles technologies dans un contexte de dictature vieillissante. Face à ce panorama, et dotés d'une bonne connaissance de l'Histoire de l'Espagne et de l'Histoire de l'art, y compris des avant-gardes, accompagné d'un esprit critique en ce qui concerne la situation politique et sociale, Equipo Crónica jette un nouveau regard sur le pays, l'art, la situation politique et le rôle de l'artiste dans cette société. Les deux peintres vont créer pour cela un nouveau code pictural qui, à partir de 1966, avec la composition Latin Lover où apparaît la couleur, les teintes planes et bon nombre des recours postérieurs va enrichir leur langage qu'ils mettront au service de la critique politique pour dénoncer la violence institutionnalisée et le pouvoir dans des séries comme La recuperación (1967-1969) ou Policía y Cultura, de 1971.

31 Outre les recours habituels du Pop Art comme les teintes planes, l'utilisation d'images empruntées aux moyens de communication ou à d'autres supports visuels de la culture de masse (cinéma, bande dessinée...), Equipo Crónica va, comme nous l'avons déjà signalé, abondamment utiliser l'histoire de la peinture et les avant-gardes du XXème siècle pour élaborer son langage plastique et focaliser son attention sur des thèmes phares tels la violence institutionnalisée ou le pouvoir.

32 L'introduction du concept de séries, apparu dès 1967 avec la réalisation d'une série de toiles réunies sous le titre de La recuperación va permettre aux deux peintres valenciens de réaliser des variations sur un même thème et d'imprimer à leur tableau une charge culturelle, politique ou sociale encore plus conforme à la situation espagnole. A partir de 
cette date donc, la trajectoire d'Equipo Crónica prend une nouvelle direction aussi bien au niveau esthétique qu'idéologique.

Que doit-on entendre par série et pourquoi ce choix ? La première raison est qu'il est difficile d'embrasser la réalité sociale culturelle ou politique extrêmement complexe de l'Espagne des années 60 en une seule œuvre. La seconde est en rapport avec la censure qui n'autorise pas de critique directe. Ainsi, pour donner corps à un discours critique virulent, Solbés et Valdés vont faire éclater les limites de l'œuvre unique en présentant divers points de vue et autres variations sur une problématique identique. Certes, l'histoire de l'art est riche en variations sur ce que Cézanne appelait le motif, mais Equipo Crónica systématise ce procédé. Grâce à des éléments iconographiques qui proviennent de diverses sources, citations chaque fois plus nombreuses d'autres artistes, les deux peintres offrent une lecture d'ensemble centrée sur un thème et, parallèlement, de multiples niveaux de lecture. Les images se caractérisent par leur polysémie et la trame des relations qui s'établissent entre elles, d'autant plus que les séries ne correspondent jamais à des unités fermées et fixes ; elles se répondent et se prolongent à travers diverses évolutions.

Une série est un ensemble d'œuvres, principalement des toiles, mais dans laquelle on inclut aussi des sculptures, des dessins, des sérigraphies et des multiples qui ont une unité chronologique (l'ensemble est peint à l'unisson durant une période de temps qui avoisine un an), iconographique (on cite un même auteur ou un même mouvement) et thématique (toutes les oeuvres développent un thème unique).

Certes, toutes ces caractéristiques peuvent être présentes dans la production de n'importe quel peintre, mais ce qui différencie les séries d'Equipo Crónica c'est leur caractère concret et fermé et leur unité thématique. La série n'est pas une façon de regrouper postérieurement des oeuvres qui ont un fort lien d'affinité. Au contraire, la série est conçue par Equipo Crónica comme série en tant que telle. Son début et sa fin ont des limites aussi distinctes que ses figures et ses couleurs. Le processus de gestation d'une série suit certaines étapes générales, bien que chaque série ait sa propre spécificité.

Le premier pas est le choix d'un thème. Et chaque série naît à partir d'un thème bien précis. Ce dernier est généralement suggéré par un événement important. La nouvelle de cet événement amène les deux peintres à une réflexion plus large sur les raisons, les causes et les effets qui l'ont produit. Parfois, il ne s'agira pas d'un événement extérieur mais d'un thème ou d'un élément tiré du quotidien comme dans les séries El billar ou Paisaje urbano. Mais, même si l'argument de la série ne provient pas d'un événement social, politique ou historique donné, des faits ou des personnages de l'actualité se glissent constamment dans les toiles.

A travers ces séries quelques caractéristiques essentielles s'affirment, notamment celles d'une iconographie empruntée aux moyens de communication ou à l'Histoire de l'art, à la peinture classique espagnole ou aux avant-gardes, toujours en ayant recours à un langage formel pop art.

Les séries possèdent leur propre autonomie, mais elles sont toujours sous-tendues par une double problématique :quelle est la relation entre l'art et la société ? Qu'est-ce que la peinture?

Plus encore, chaque série aborde des aspects qui ont trait à la fonction sociale de l'art, à la production artistique comme production culturelle, à la culture comme instrument de libération ou de répression. Ce premier niveau entre généralement en connexion avec des 
allusions à des événements concrets de la réalité historique du moment : les fusillades de février 1975, la mort de Franco, le retour du Guernica en Espagne. De la même façon, leur questionnement sur la peinture n'est jamais indépendant de la question sur leur propre peinture, sur leur trajectoire picturale si particulière. Et tous ces niveaux fonctionnent en interdépendance.

En effet, jusqu'à présent, chacune des toiles représentées abordaient un thème particulier, traité selon un procédé bien déterminé. Or, à partir de l'apparition des séries, le thème développé ne le sera plus à partir d'une seule œuvre mais à partir d'un nombre déterminé de toiles. Ce qui signifie que le sens, que le message ne sera plus circonscrit à une toile en particulier mais inscrit dans une suite de toiles. Le sens n'est plus limité à un tableau, il se développe à partir et autour d'une série de tableaux, ce qui fait éclater le message narratif, comme si chacune des compositions devenait l'élément, la pièce d'un puzzle qu'il appartiendrait au spectateur de réunir pour parvenir au message esthétique et idéologique véhiculé par l'ensemble.

Chaque série, de plus, aborde, en règle générale, un thème différent. Cependant, des thèmes complémentaires, des prolongements en écho, des renvois existent ou se produisent, à tel point que le spectateur ne sait plus où se situent précisément les limites du récit qu'Equipo Crónica construit et déconstruit à l'envi.

La série Autopsia de un oficio, par exemple, suppose principalement, à travers la citation des Ménines de Velázquez comme référent iconique, une réflexion sur la pratique artistique à un moment de crise généralisée de l'art en tant qu'arme contre le pouvoir. Le titre même de l'ensemble affirme la mort de la pratique artistique dont le pouvoir de sédition est condamné au néant. Policia y cultura, pour sa part, offre une critique de la violence institutionnalisée. Peinte en un laps de temps très réduit (automne 70-printemps 71), cette série apparaît comme l'un des projets les plus ambitieux d'Equipo Crónica. Il s'agit d'un ensemble de 13 œuvres de même format (200x200) où les deux peintres, en une sorte de révision systématique de leur période d'apprentissage, déploient tous les recours picturaux utilisés -ou partiellement envisagés- antérieurement. La série suggère une disposition narrative, mais en réalité on observe qu'il n'y a pas de narration à proprement parler puisqu'il n'y a pas d'ordre ni de séquences, même à l'intérieur de chacune des scènes représentées. En réalité, il s'agit d'une seule scène, toujours la même, mais différente à chaque fois. Il n'y a donc pas dans cette série, contrairement aux œuvres de la période initiale, de lecture possible, parce qu'il y a absence de linéarité, ce qui concourt à une certaine ambiguïté, d'autant que les associations d'images sont souvent extrêmement inattendues. Rafael Solbés alla même jusqu'à qualifier la série de «barroquismo compositivo ", faisant probablement référence à la complexité spatiale des scènes, un espace purement artificiel, non pas représentatif mais construit. Or l'exploitation de ce type d'espace pictural fragmenté, complexe, artificiel, né de la simultanéité et de la juxtaposition de hiérarchies différentes deviendra une constante formelle de toute l'œuvre d'Equipo Crónica.

43 Si la série Policia y Cultura apparaît comme le moment culminant de la période d'apprentissage d'un nouveau langage pictural apte à interroger l'art et à critiquer la réalité sociale, politique et historique de son temps, la série de 25 toiles intitulée Serie negra réalisée entre 1971 et 1972 est le premier témoignage de la maturité de leurs pensées artistiques. Le thème de la série est celui de la peinture en tant que discipline. Toutefois, ce thème ne se présente pas de façon directe mais à travers une suite de métaphores qui s'articulent autour d'un élément de la culture populaire : le genre noir. 
Ici, les références à la réalité politique et sociale se font moins évidentes quand elles ne disparaissent pas purement et simplement. En revanche, les références à la peinture comme technique et comme métier émergent de façon beaucoup plus nette que dans les séries antérieures. Trois types d'éléments sont mis en relation dans chacune des toiles. Tout d'abord des photogrammes empruntés au cinéma classique du genre noir qui occupent le centre de la composition. Ensuite, des images empruntées à la peinture, principalement aux avant-gardes du XXème siècle: Lichtenstein, Hopper, Leger, de Chirico, Kandinsky, Ernst... Enfin, le dernier élément présent, présenté, représenté conduit pour sa part à un autre type de considérations puisqu'il s'agit d'encrier, de crayons de couleurs, de cahier d'écolier, de tubes de peinture, de règles, en un mot d'un ensemble d'outils réservés à l'apprentissage non seulement des enfants mais également des étudiants d'art, Ces instruments ont été peints en trompe l'œil pour leur faire occuper un plan situé devant le reste de la composition comme si on avait voulu les rendre indépendants dans le but de laisser croire qu'ils reposent sur une superficie transparente imaginaire, tel du verre. A partir de ces trois éléments, Valdés et Solbés proposent une méditation multiple sur l'apprentissage et les années d'enseignement -qui étaient d'ailleurs celles durant lesquels on projetait des films de gangsters en noir et blanc-, la peinture et sa confrontation avec le cinéma, la violence des scènes comme compagne du savoir et de la création picturale.

Le langage de cette série est peut-être moins spectaculaire que celui utilisé dans Policia y cultura, mais en revanche, Solbés et Valdés introduisent des innovations linguistiques importantes comme, par exemple, le recours au trompe l'œil qui augmente la complexité de la perception sans pour autant augmenter la complexité des formes Mais la caractéristique la plus significative est l'introduction systématique d'éléments autobiographiques. Ces références biographiques fictives tissent un plan continu de sens qui entrent en contact avec le processus de construction du sujet artiste qui est au cœur de la réflexion d'Equipo Crónica.

D'ailleurs, à partir de la réalisation de cette série, à de rares exceptions près, la réflexion des deux peintres valenciens portera essentiellement sur la nature de la peinture ellemême, sur la façon de l'articuler ou d' en explorer certains aspects. Et ce même dans les séries où, comme on peut l'observer dans El paredón, les événements politiques occupent le premier plan. Rien d'étonnant dès lors à ce que certaines d'entre elles comme Retratos bodegones y paisajes présentent une double problématique : celle du traitement des genres en peinture et celle de la tradition artistique espagnole.

Mais c'est sans doute la série La trama qui offre l'exemple le plus significatif du jeu qu'opèrent Solbés et Valdés quant à l'élaboration du sens et aux possibilités extrêmes qu'offre la peinture puisque, dans ce cas précis, il ne s'agit pas d'une série composée de plusieurs toiles, mais d'une série de plusieurs triptyques, ce qui en rend la lecture beaucoup plus complexe, poussant les limites de l'interprétation à un niveau extrême, aussi bien d'un point de vue plastique que d'un point de vue conceptuel.

Réalisées à la fin de la dictature franquiste quand les medias remémoraient la vie du Caudillo, les 12 toiles de la série -qui accuse une structure identique- mettent en relation, en réseau, toutes une série d'éléments qui vont interférer les uns avec les autres pour produire du sens. Ici, les relations multiples qui s'opèrent passent d'un tableau à l'autre. L'argument narratif, comme le précise Michèle Dalmace, repose sur une scène représentée sur le plus grand des panneaux du triptyque, scène qui se répète comme sous l'effet d'un zoom dans le tableau où se trouve le spectateur. Dans ce panneau également, 
cette scène est à nouveau incluse, mise en abyme, mais peinte avec le style d'origine en format plus petit. Le spectateur de dos qui fait d'abord écran avant de faire philtre sert pour relier les différents éléments. Cependant, la scène non seulement est empruntée à un peintre, en ce qui concerne le thème du triptyque et sa composition mais elle est également peinte avec le style d'un autre peintre. Par conséquent, chaque triptyque a comme élément de base un couple stylistique: Solana / Malevitch, Bacon/Gris, Lichtenstein /Hopper, Daumier / De Chirico, Picasso/ Klimt El Lissinsky/ Miro... Ainsi, la citation d'un artiste est peinte selon le style d'un autre. Hopper est vu à travers le style de Lichtenstein, Klimt par Picasso et ainsi de suite. On aboutit alors à un réseau aux combinaisons multiples qui révèlent la grande complexité de la série où se croisent et interfèrent toujours Histoire et production artistique. En effet, on met en relation cette double citation iconographique avec une référence photographique des Archives de Franco, puisque la série propose des scènes de la vie du Généralissime. Il ne s'agit pas, cette fois-ci, de documents provenant des moyens de communication mais de matériel d'archives qui furent publiés après la mort de Franco. Dans les triptyques, une photo, toujours située sur la partie supérieure du plus grand des panneaux, sert de référence et de constante tandis qu'un acte officiel écrit occupe intégralement le panneau le plus petit.

Dans El Africa, le spectateur qui observe une partie de l'œuvre sert de philtre entre le fragment de scène tiré de Judith et Holopherne de Klimt, mais peint à la manière cubiste de Picasso et la scène complète du panneau le plus grand à travers la même image Klimt/ Picasso qui se répète; il sert également de philtre entre cette représentation et la scène d'origine -de Klimt- qui se trouve dans un format réduit, dans l'angle supérieur. De plus, par réminiscence visuelle, l'aspect de la scène d'origine dans un cadre renvoie à la photographie biographique de Franco dans le tableau le plus grand. Cette dernière, photographie tirée d'archives, est, à son tour, mise en relation avec une citation textuelle ou iconologique qui correspond à un document officiel de caractère biographique -la blessure de guerre, l'emblème de la Phalange, le texte du referendum- qui occupe tout l'espace du troisième panneau.

De plus, Valdés et Solbés, comme ils l'ont déjà précédemment pratiqué, ont introduit un intrus dans le tableau. Il ne s'agit pas, cette fois-ci, d'un élément qui indique une rupture ou un changement soudain dans la répétition d'une image comme dans América America, il ne s'agit pas non plus de l'intrus qui apparaît dans le tableau qui porte ce nom et qui renvoie à un personnage tiré d'un autre genre, ce n'est pas non plus la référence à un peintre d'une autre époque installé entre des peintres contemporains mais d'un nouveau type d'intrus sans lien apparent avec l'argument narratif : des notes de musique, un tube de peinture, des cigarettes qui envahissent la scène principale et le tableau qui sert de référence, créant ainsi un obstacle entre l'œuvre et son spectateur, ou marquant une sorte de limite afin d'empêcher l'adhésion spontanée du spectateur au discours. Les deux peintres valenciens devaient expliquer que « estos elementos repartidos en la superficie de los cuadros 1 y 3 pretenden romper con la estructura formal y temática de los cuadros al ser utilizados como en el surrealismo automático. »

Et, logiquement, la seule toile dans laquelle ces éléments n'apparaissent pas est celle où se trouve le spectateur puisque ce dernier sert en quelque sorte de point d'intersection entre le tableau et le spectateur réel qui est hors du triptyque.

51 On le constate aisément, les limites du récit semblent être illimitées et l'on pourrait même aller jusqu'à affirmer que dans la peinture d'Equipo Crónica il n'existe point de limites quant au traitement de l'image et à ses implications idéologiques et esthétiques. 
Les éléments se disloquent, se répètent, se prolongent, se fragmentent, s'échappent même des toiles comme dans El museo ou La visita 1 et 2 de la série Guernica, se superposent, se juxtaposent, se déclinent et se combinent selon des modalités diverses à l'intérieur d'un même tableau ou d'une même série.

Dans le triptyque intitulé Sorolla como pretexto qui n'est issu d'aucune série mais qui appartient à un ensemble d'œuvres réalisées entre 1974 et 1975, les deux artistes valenciens empruntent une image topique peinte par Joaquin Sorolla: la plage de La Malvarosa. Ils la représentent deux fois dans les deux premiers panneaux du triptyque. Dans celui de gauche, ils la réalisent comme si elle avait été dessinée à l'aide de crayons de couleurs; surgit également, avec un effet de relief, dans l'angle inférieur droit du panneau, un crayon rouge.

Dans le panneau central, la même image est répétée, mais cette fois elle semble avoir été peinte avec des pinceaux et à l'acrylique. Dans l'angle inférieur droit, parallèlement au crayon rouge, surgit également un instrument producteur d'images: un pinceau. Le troisième panneau propose une image différente : un plan de la plage de La Malvarosa (avec le nom répété trois fois) et à nouveau depuis le coin inférieur droit, de façon parallèle aux deux autres éléments déjà mentionnés, apparaît un tire-ligne, supposé être responsable du plan. Sur celui-ci a été peinte une photographie comme fixée avec une punaise juste au-dessus de la ligne de plage qui montre un motif semblable à celui des deux autres panneaux. : des enfants en train de jouer au bord de l'eau tandis que les jambes d'un quatrième enfant à peine entrevu semblent s'être enfoncée dans l'eau.

Ce qui est ici mis en jeu et livré à la méditation, ce sont les formes de la représentation visuelle et les possibilités illimitées de représentation d'un même motif. Des enfants jouant sur la plage de La Malvarosa peuvent être dessinés au crayon, peints à l'acrylique ou bien, dans un jeu conceptuel, on peut représenter les enfants en train de jouer à l'aide d'une photographie que l'on place sur un plan qui représente La Malvarosa. Jeu sur la peinture et la représentation un peu dans la lignée de Ceci n'est pas une pipe de Magritte pour argumenter que ceci n'est pas un Sorolla, ni la plage de La Malvarosa mais que nous peintres dessinons ou peignons la plage qu'a peint Sorolla et nous la mettons à côté de la plage réelle que nous peignons aussi mais à travers la représentation d'un plan et d'une photo...

Si,comme nous venons de l'observer, Equipo Crónica s'est livré à une transgression des codes traditionnels de la peinture afin de mettre en lumière une idéologie qui, au début, visait principalement à une critique sociale politique et historique, si cette peinture, novatrice, innovante par bien des aspects, révolutionnaire même à bien des égards, a été mise au service d'une pensée militante, très rapidement la déconstruction du code établi,la transgression des dogmes académiques a évolué vers une réflexion en profondeur sur l'art. L'éclatement des limites du langage se donnait également comme intention une réflexion esthétique qui allait mettre l'artiste et l'art au centre et au cœur des préoccupations idéologiques des deux peintres valenciens. Peinture métapicturale donc qui à partir de techniques formelles rénovées, poussées à l'extrême, invite à lire les toiles non pas exclusivement comme des chroniques sociales mais comme de véritables manifestes esthétiques qui abordent des questions aussi variées -mais non moins fondamentales- que la couleur, les supports, les techniques, les codes, le regard, les genres, l'Histoire de l'art mais également la fonction de la peinture au sein de la société, le rôle de l'artiste et l'art comme activité culturelle. 

de couleurs, règle, équerre...On les retrouve tout au long des séries et, la plupart du temps, ils produisent des effets surprenants, ils favorisent l'ironie, ils font allusion à l'apprentissage académique des Beaux Arts mais, dans le même temps, leur présence tend à désacraliser la peinture en tant qu'art ainsi que les outils traditionnels du peintre. D'autres fois, ils constituent une mise en abyme ou bien ils forment un ensemble sémantiquement signifiant comme dans le tableau El dia que aprendí a escribir con tinta (1972). De même, ces objets qui font partie du quotidien du peintre peuvent se transformer en éléments agressifs comme dans La violacion de la regla où Pintar es como golpear ou représentatifs de la transgression de la norme.. Mais au-delà des normes, ils peuvent symboliser la difficulté pour certaine classe sociale d'accéder à la culture comme dans la série El billar (1977). Comme l'explique Michèle Dalmace, « répartis tout au long des séries, les objets convergent, enrichissent, nuancent, une réflexion autour de l'apprentissage, des conventions et de la pratique artistique qu'ils désacralisent. De fait, ils assurent la continuité du discours ${ }^{4}$. »

$59 \mathrm{Au}$ terme de cette analyse -certes nécessairement réduite, mais non réductrice- on retiendra que la trajectoire picturale de Solbés et Valdés s'inscrit dans une perspective résolument subversive qui vise à dépasser les limites non seulement des techniques formelles traditionnelles mais également du discours iconique aseptisé des années dite de l'après-guerre.

60 S'écartant des modèles conventionnels pour faire entrer dans leurs toiles des recours stylistiques hérités du pop art mais aussi empruntés à l'histoire de l'art et aux supports culturels de masse de l'époque, Equipo Crónica s'engage résolument dans la voie de la critique sociale, historique et politique d'une période trouble. Pour autant, cette peinture militante ne se cantonne pas exclusivement à faire œuvre de témoignage ou de reportage. Bien au contraire, au lieu d'atteindre ses limites, le récit iconique des deux peintres valenciens, au fil du temps et au fil des séries, évolue vers une réflexion sur la peinture elle-même, puisque leurs tableaux se donnent à lire comme un questionnement sans cesse répété sur la fonction de l'art, sur la nature de l'image et sa possible manipulation, sur son impact et son pouvoir même sans oublier la figure de l'artiste dont l'image est fréquemment démythifiée.

61 Au début des années 80 , quand le Collectif disparaît à la suite du décès de Solbés, d'autres acteurs de la vie artistique et culturelle espagnole allaient emprunter, dans d'autres 
domaines, notamment celui du cinéma, la même voie. Comment ne pas penser dès lors à Pedro Almodovar?

\section{BIBLIOGRAPHIE}

BARRAL I ALTET, Xavier, L'art espagnol, Paris, Bordas, 1996.

EQUIPO CRÓNICA, Catalogo razonado a cargo de Michèle Dalmace, Valencia, IVAM, 2001.

EQUIPO CRÓNICA, Saint-Etienne, Maison de la Culture, 1975.

LA NOUVELLE FIGURATION, Paris, Centre Georges Pompidou, 2008.

LLORENS, Tomas, Equipo Crónica, Barcelona, Gili, 1972.

MARIN, Ricardo, Equipo Crónica : pintura, cultura, sociedad, Valence, Institucion Alfonso el

Magnánimo, 2001.

MARIN, Ricardo, El realismo social en la plástica valenciana 1964-1975, Valencia, Nau Llibres, 1981.

Certaines reproductions des tableaux évoqués dans cet article peuvent être consultés sur le site http://www.ciudadpintura.com/

\section{NOTES}

1. VID. La emigración, 1964, La huelga, 1965 ; Manifestación, 1965 ; Vietnam, 1965 ; Bomba atómica, 1965.

2. VID. El recinto, 1969 ; El alambique, 1969 ; El ejecutivo, 1969 ; Las estructuras cambian las esencias permanentes, 1969; El Bosco en la oficina, 1969.

3. VID. Las reglas del juego, 1972 ; Bodegón nacional, 1972 ; Vallecas Melody, 1972.

4. Cf. Equipo Crónica, Catalogo razonado a cargo de Michèle Dalmace, Valencia, IVAM, 2001, p. 77.

\section{RÉSUMÉS}

Cette communication consacrée aux peintres valenciens Rafael Solbés et Manolo Valdés, plus connus sous le nom d'Equipo Crónica propose une réflexion sur le nouveau langage pictural mis en œuvre, au milieu des années 60, par les deux artistes dans des toiles dont les codes iconiques dépassent les limites, voire les limitations, d'une peinture alors engoncée dans un certain académisme afin de porter un regard critique sur la société espagnole du franquisme finissant. 
Réflexion sur la transgression des limites, sur la rénovation de l'écriture picturale, mais également sur la création artistique, sur la pratique de la peinture, sur la fonction de l'art et sur les rapports que ce dernier entretient avec la société puisque les pratiques esthétiques opérées par ce que certains appellent la Nouvelle Figuration entraînent généralement un véritable questionnement sur la peinture elle-même.

Ese trabajo dedicado a los pintores valencianos Rafael Solbés y Manolo Valdés, más conocidos como Equipo Crónica, propone una reflexión acerca del nuevo lenguaje pictórico puesto en obra, a mediados de los años 60, por ambos artistas en cuadros en los que los códigos de la pintura superan los límites, hasta las limitaciones, de una pintura hasta ahora estancada en cierto academismo formal con tal de echar una mirada crítica sobre la sociedad española de la época franquista. Reflexión acerca de la transgresión de los límites, acerca también de la renovación de la escritura pictorica, pero asimismo reflexión acerca de la creación artística, del papel del arte y de las relaciones que entreteje con la sociedad ya que las prácticas estéticas de lo que unos llaman la Nueva Figuración conducen en general a un verdadero cuestionamiento acerca de la pintura misma.

INDEX

Mots-clés : peinture, limites, Nouvelle figuration, Equipo Crónica

Index chronologique : XXe siècle

\section{AUTEUR}

\section{FABRICE PARISOT}

Université de Nice Sophia-Antipolis, CIRCPLES, EA 3159 